\title{
Optic flow induced nystagmus
}

\author{
A Petzold, G T Plant
}

J Neurol Neurosurg Psychiatry 2005;76:1 173-1174. doi: 10.1136/jnnp.2004.052720

A new form of nystagmus is described in this paper: optic flow induced nystagmus (OFN). Optic field flow, for example when driving or viewing films on a large screen, triggered non-fatiguable upbeat nystagmus. This type of nystagmus could not be induced by vestibular stimuli, positioning, or self motion. The aetiology and anatomical correlate of OFN remains unknown. Treatment with gabapentin suppressed OFN.

$\mathrm{H}$ ere we describe for the first time a new form of paroxysmal upbeat nystagmus triggered by optic flow stimulation. A 42 year old female patient complained of paroxysmal vertical oscillopsia, particularly after driving a vehicle. She had always been aware of "ghosting" of images before the onset of oscillopsia. Eye closure at an early stage would sometimes prevent the onset of an attack. She described the oscillopsia as similar to the loss of the "vertical hold" on a television. Just lying down would not bring about resolution, and attacks would last until she went to sleep. There were no other features. This phenomenon could also be triggered by tiredness, stress, emotional factors, or a visit to the cinema. She had suffered from these attacks for as long as she could remember. She had found out empirically that four to five tablets of coproxamol (dextropropoxyphene combined with paracetamol) delayed the onset of oscillopsia, for example, while driving. She had undergone eye occlusion therapy as a child and had squint surgery at the ages of 2.5 and 16 years. She is a non-smoker and does not drink alcohol.

Examination revealed amblyopia of the left eye and the patient could only perceive shapes and strong colour contrasts on this side. The visual acuity in the right eye was 6/6 (20/20 Snellen equivalent), with full colour vision (17/17 Ishihara charts). On cover test, a slight left convergent strabismus and a left hypertropia in all gaze positions were noted. She did not take up fixation with the left eye. Tests revealed absence of binocular function with suppression of the left eye. The eye movements were normal, as was the remainder of the neurological and neuro-ophthalmological examination; in particular, there was no direct ophthalmoscopic evidence for vertical nystagmus between attacks. Nystagmus was not triggered by positional testing, head movements, neck extension, or convergence. The optokinetic nystagmus response to horizontal stimulation was normal, and covering either eye did not reveal latent nystagmus. Blood tests and standard EEG recorded between attacks were normal. The MRI demonstrated a minor Arnold-Chiari type I malformation (fig 1A) without compression of the cervicomedullary junction.

Because we were not able to trigger the nystagmus in clinic, we asked her to visit the cinema before the next visit. After she viewed a film on a 1: 15 large screen presentation at an IMAX cinema, we witnessed and videotaped the nonfatiguable upbeat nystagmus ( see video online). Although we

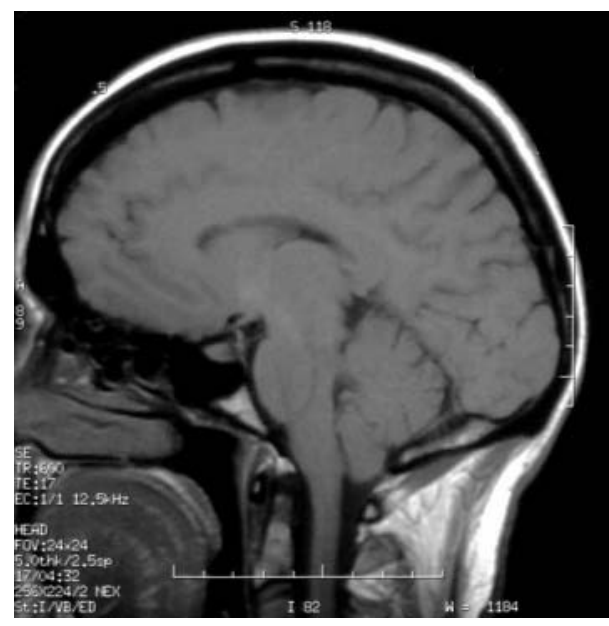

Figure $1 \mathrm{MRI}$ of the head of this 42 year old woman with optic flow induced nystagmus. The sagittal section shows a minor Arnold-Chiari type I malformation. There is no significant compression of the medulla.

were not able to record the eye movements formally, it appears from the video recording that the waveform was of runaway/increasing velocity. The ghosting of images noticed before the onset of nystagmus is explained by the $2-3 \mathrm{~mm}$ left hypertropia either because the deviation increased or because of failure of suppression. She was treated successfully with gabapentin $300 \mathrm{mg} /$ day but the symptoms tended to recur until the daily dose reached $1500 \mathrm{mg} /$ day. On this dose she became stable and asymptomatic.

\section{DISCUSSION}

Upbeat nystagmus has been reported with lesions in various locations. It may be permanent or transient, and can only be suppressed poorly by fixation. ${ }^{12}$ Transient or paroxysmal upbeat nystagmus can be found in patients exposed to toxic metabolites including tobacco, barbiturates, and organophosphates. It occurs classically in Wernicke's encephalopathy and in epilepsy. ${ }^{3}$ Paroxysmal nystagmus can be positional, and anecdotally has been observed in middle ear infections and in vestibular disorders. ${ }^{2}$ Otolith function can influence vertical nystagmus. ${ }^{4}$ This can be demonstrated clinically by positioning or convergence, but in this patient these manoeuvres did not modify the nystagmus. An important cause of latent nystagmus is congenital strabismus. ${ }^{5}$ Although we were not able to demonstrate latent nystagmus, the presence of congenital strabismus in our patient might be of functional relevance. Paroxysmal nystagmus can result from pathology around the foramen magnum, such as an Arnold-Chiari malformation, which can be triggered by neck flexion and extension. ${ }^{6}$ Because neck flexion did not trigger the onset of nystagmus and the cervicomedullary junction

Abbreviations: OFN, optic flow induced nystagmus 
did not appear on MRI to be compressed (fig 1), we were not sure whether the Arnold-Chiari malformation was of aetiological significance.

The term optic flow was coined by Gibson in a series of seminal papers on visual perception in the 1960s. ${ }^{7}$ Optic flow is a physiological phenomenon that helps a person to extract visual characteristics from the two dimensional image flow on the retina while moving through a three dimensional environment. Simplified, there are two kinds of optic flow: rotational and translational. During pure translation, the optic flow consists of a stream of images emerging from a focus of expansion straight ahead and disappearing into a focus of contraction behind. This optic flow field provides information about self motion, direction of heading, depth, and time to collision. During self movement, the optic flow causes an optokinetic stimulation that is strongest in the nasal to temporal direction horizontally, and usually downward, the ground being closer than the sky. ${ }^{8}$ Compensatory eye movements during forward motion (centrifugal flow) thus have a downward component. We speculate that decompensation of signal processing in the CNS may have been the cause of the upbeat nystagmus in the present case, and that a component of vestibular mismatch was also required, as optic flow nystagmus was not induced by self motion such as gait.

It would be interesting to investigate in more detail the stimuli required to induce nystagmus. Watching television in a dark room (approximately 10 degrees of visual angle) did not induce nystagmus, therefore we suspect whole field stimulation would be needed. Investigating the mechanisms triggering the nystagmus, perhaps in combination with high resolution functional imaging techniques and eye movement recordings, might provide a potential anatomical model in future studies. A candidate region for optic flow extraction would be the medial superior temporal area, also known as V5a. ${ }^{9}$ However, at the present time all that we can say is that the minimal stimulus required to produce nystagmus seems to be optic flow stimulation of the peripheral retina, the threshold of which might be reduced by tiredness, stress, and emotional factors. Understandably, our patient has declined our invitation to discontinue gabapentin for further studies.

Treatment with high dose gabapentin was dramatically effective, probably by increasing GABA levels in the human brain due to stimulation of glutamic acid decarboxylase and inhibition of GABA transaminase. ${ }^{2}{ }^{10}$ It has been proposed that this results in stabilisation of the neuronal integrator. ${ }^{2}{ }^{10}$ The partial response to coproxamol experienced by this patient was probably due to the opiate, dextropropoxyphene.
We do not believe this is the result of a mere placebo effect, but acknowledge that this single report does not provide conclusive evidence for the effectiveness of gabapentin in this type of nystagmus.

In conclusion, we are uncertain whether the nystagmus is congenital in type (because it is a lifelong phenomenon and amblyopia was present) or whether it is related to the Arnold Chiari malformation. We would be interested to hear of any other examples of nystagmus provoked by optic flow phenomena and we propose to name this form of nystagmus optic flow induced nystagmus (OFN).

\section{ACKNOWLEDGEMENTS}

We should like to thank Professors R J Leigh and J Sharpe for their comments on the manuscript.

\section{Authors' affiliations}

A Petzold, Institute of Neurology, London, UK

A Petzold, G T Plant, National Hospital of Neurology and

Neurosurgery, and Moorfields Eye Hospital, London, UK

Competing interests: none declared

Correspondence to: $\operatorname{Dr}$ A Petzold, Institute of Neurology and Neuroimmunology, Queen Square, London WCIN 3BG, UK; a.petzold@ion.ucl.ac.uk

Received 26 August 2004

In revised form 21 December 2004

Accepted 21 December 2004

\section{REFERENCES}

1 Serra A, Leigh R. Diagnostic value of nystagmus: spontaneous and induced ocular oscillations. J Neurol Neurosurg Psychiatry 2002;73:608-15.

2 In: Leigh R, Zee D, eds. The neurology of eye movements, 3rd ed. Oxford: Oxford University Press, 1998

3 Gire C, Somma-Mauvais H, Nicaise C, et al. Epileptic nystagmus: electroclinical study of a case. Epileptic Disord 2001;3:33-7.

4 Fisher A, Gresty M, Chambers B, et al. Primary position upbeating nystagmus. A variety of central positional nystagmus. Brain 1983;106:949-64.

5 Gresty M, Metcalfe T, Timms C, et al. Neurology of latent nystagmus. Brain 1992; 115:1303-21.

6 Mossman S, Bronstein A, Gresty M, et al. Convergence nystagmus associated with Arnold-Chiari malformation. Arch Neurol 1990;47:303-57.

7 Gibson J. The visual perception of objective motion and subjective movement. Psychol Rev 1954;61:304-14.

8 Garbutt S, Han Y, Kumar A, et al. Disorders of vertical optokinetic nystagmus in patients with ocular misalignment. Vision Res 2003:43:347-57.

9 Greenlee M. Human cortical areas underlying the perception of optic flow: brain imaging studies. Int Rev Neurobiol 2000;44:269-92.

10 Stahl J, Plant G, Leigh R. Medical treatment of nystagmus and its visual consequences. J R Soc Med 2002;95:235-7. 